\title{
'Negative' surface differential rotation in stars having low Coriolis numbers (slow rotation or high turbulence)
}

\author{
Kwing L. Chan ${ }^{1}$ \\ ${ }^{1}$ Department of Mathematics, \\ Hong Kong University of Science and Technology, Hong Kong, China \\ email: maklchan@ust.hk
}

\begin{abstract}
A general picture of differential rotation in cool stars is that they are 'solar-like', with the equator spinning faster than the poles. Such surface differential rotation profiles have also been demonstrated by some three-dimensional simulations. In our numerical investigation of rotating convection (both regional and global), we found that this picture is not universally applicable. The equator may spin substantially slower than the poles $\left(\left(\Omega_{\text {equator }}-\Omega_{\text {pole }}\right) / \Omega\right.$ can reach $-50 \%)$. The key parameter that determines the transition in behavior is the Coriolis number (inverse Rossby number). 'Negative' differential rotation of the equator (relative to the mean rotation) occurs if the Coriolis number is below a critical value.
\end{abstract}

Keywords. stars: interiors, rotation; convection

\section{Introduction}

The interaction of convection and rotation can produce different zonal flow patterns. A switch in the sign of the vertical shear in the zonal flow at the equator has been discussed in an earlier conference paper (Chan 2003). The pattern transition was linked to the magnitude of the Coriolis parameter (inverse of the Rossby number). The process was demonstrated by both regional f-box calculations and global-scale spectral calculations. In recent years, 'anti-solar' differential rotations have been reported in a number of observations (Kovain et al. 2007, Erdem et al. 2009) and models (Kitchantinov \& Rudiger 2004, Steffen \& Freytag 2007, Palacios \& Brun 2007). The phenomenon, we believe, reflects the situation of low Coriolis number (high Rossby number) as discussed in the earlier paper. In this paper, we present a more systematic (and higher resolution) investigation of the flow field transition in a global-scale shell model.

\section{Model}

The model solves the stratified form of the compressible hydrodynamics equations (Chan 1994) for an ideal gas in a rotating convection zone with a thickness of $30 \%$ outer radius. The stratification contains 6.3 pressure scale heights. Four calculations with different values of angular velocity $\Omega$ have been made. The rest of the model setup is not changed. Values of the Coriolis number $(\mathrm{Co}=\Omega d / v)$ for the four cases are 4.6, 9.6, 22 , and 39 . The length scale $d$ is taken to be the depth of the layer and the velocity scale $v$ is the root-mean-square total velocity in the domain. The three-dimensional numerical code uses the transform spectral method, and the spatial resolution is T84 $\times 116$ where T84 stands for triangular truncation with maximum zonal degree 84 and 116 stands for the number of vertical grid levels. The models took long time to relax. 


\section{Results}

The latitude-radius distributions of the longitudinally and temporally averaged relative angular velocity (in comparison with the mean rotation of the shell) are shown on the panels of Figure 1 in an order of increasing Co. The light/dark shadings represent positive/negative values. Figure 2 show the latitudinal profiles of the surface differential angular velocity for the cases arranged in the same order. A critical value of Co exists between 9.6 and 22. For lower Co, the radial shear of the mean zonal velocity at the equator is negative; for higher Co, the shear is positive. When the shear is positive, the surface differential rotation is 'solar-like'; when the shear has the opposite sign, the differential rotation is 'anti-solar'. Besides this qualitative change, the profiles show further variations for different Co values.

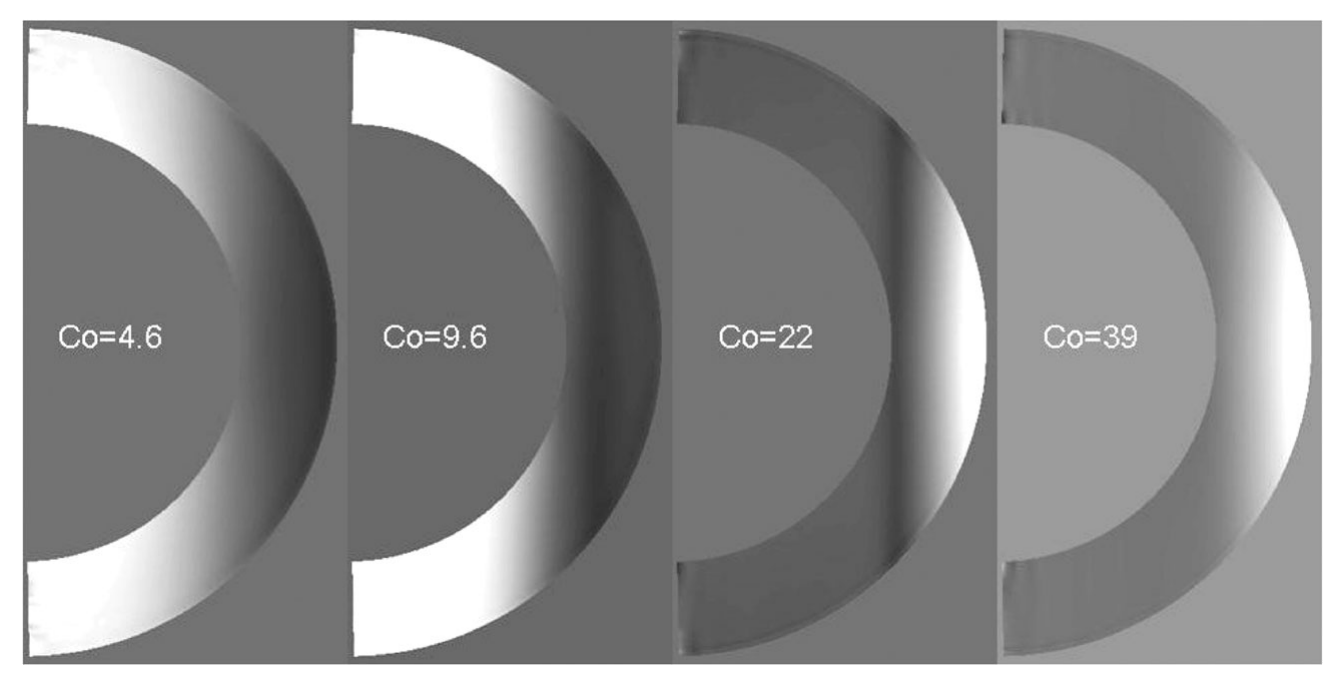

Figure 1. Differential angular velocity distributions
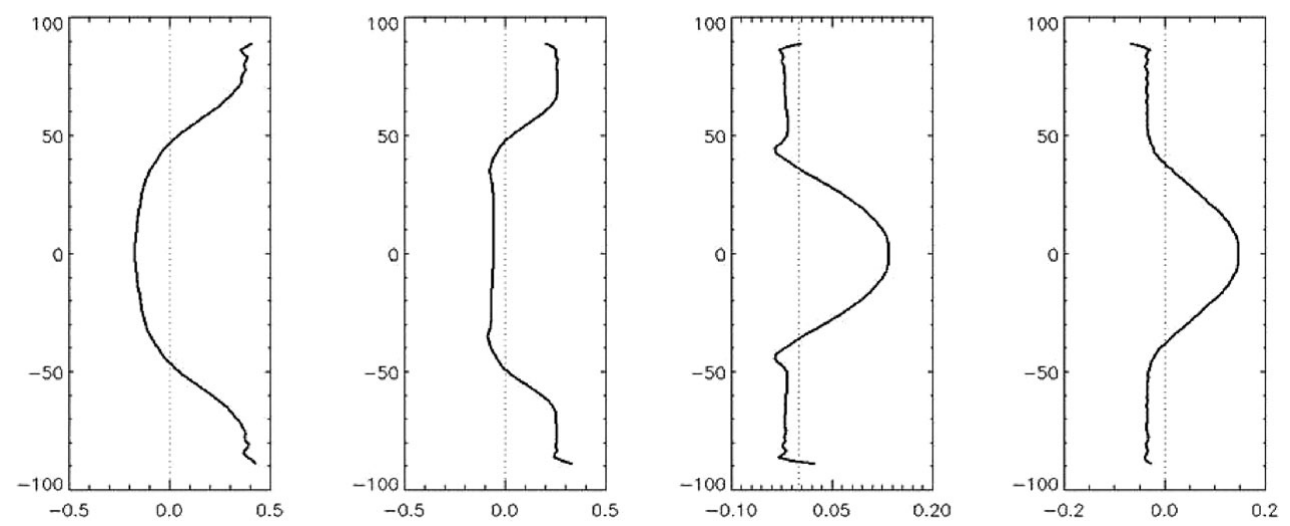

Figure 2. Latitudinal profiles of the surface differential angular velocity 


\section{Conclusion}

The Coriolis number determines whether differential rotation at the surface of a cool star is 'solar-like' or 'anti-solar'. Observation of this surface characteristic may help to impose constraints on the properties of the convection zone (e.g. depth, turbulence strength).

\section{Acknowledgement}

KLC thanks the Hong Kong Research Grants Council for support (600309).

\section{References}

Chan, K. L. 2003, in S. Turcotte, S. C. Keller \& M. Cavallo (eds.), 3D Stellar Evolution (Ann Arbor: Sheridan), p. 168 (can be found at http://www.math.ust.hk/ maklchan)

Chan, K. L., Mayr, H. G., Mengel, J. G., \& Harris, I. 1994, J. Comput. Phys., 113, 165

Erdem, A. et al. 2009, New Astron., 14, 545

Kitchatinov, L. L. \& Rudiger, G. 2004, AN, 325, 496

Palacios, A. \& Brun, A. S. 2007, AN, 328, 1114

Steffen, M. \& Freytag, B. 2007, AN, 328, 1054 